\title{
Pseudoscalar Mesons and Mixing Models
}

F. D. Gault and A. B. Rimmer

Z. Phys. C - Particles and Fields 8, 353 (1981)

The first of the three Eqs. (2.14) should read:

$|\eta\rangle=0.784|N S\rangle-0.621|S\rangle-3.7 \times 10^{-3}|c \bar{c}\rangle$.

\section{Renormalization and Short Distance Properties of String Type Equations in QCD}

H. Dorn and E. Wieczorek

Z. Phys. C - Particles and Fields 9, 49 (1981)

In the matrix (26) due to a missed diagram [the analogue to (3e) with two gluon legs] the following changes have to be made: $\gamma_{22}=\frac{g^{2}}{4 \pi^{2}} \cdot \frac{3}{8} C_{A}, \gamma_{99}=\frac{g^{2}}{4 \pi^{2}} \cdot \frac{5}{8} C_{A}$. As a consequence the operators $\Omega_{\mu \nu}^{(1)}, \Omega_{\mu \nu}^{(2)}, \Omega_{\mu \nu}^{(3)}, \Omega_{\mu \nu}^{(4)}$ renormalize among themselves. This effects the leading short distance singularity which will be discussed in a forthcoming paper. 